\title{
Mitochondrial Genome Resource of a Grapevine Strain of Trichoderma harzianum, a Potential Biological Control Agent for Fungal Canker Diseases
}

\author{
Christopher Michael Wallis, ${ }^{\dagger}(\mathbb{D})$ Jianchi Chen, and Adalberto A. Perez de Leon \\ Crop Diseases, Pests and Genetics Research Unit, USDA-ARS San Joaquin Valley Agricultural \\ Sciences Center, Parlier, CA 93648
}

\section{Resource Announcement}

Trichoderma spp. are commonly used as bioremediation agents, as biological controls, and for making biofuels. Herein, a Trichoderma harzianum strain PAR3 was isolated from grapevine roots in central California, U.S.A. As part of a larger whole genome sequencing effort, the mitochondrial genome (mitogenome) sequence was obtained for the PAR3 strain. The mitogenome is $27,631 \mathrm{bp}$, containing genes of 14 core mitochondrial proteins, 25 transfer RNAs, and two ribosomal RNAs, with a GC\% content of $27.55 \%$. A BLAST search using the PAR3 mitogenome as a query against the GenBank sequence database showed that the mitogenome MUT3171 (29,791 bp) of Trichoderma lixii was the most similar (query coverage = $99 \%$; percentage identity $=100 \%$ ) with two major deletions, $1,339 \mathrm{bp}$ and $821 \mathrm{bp}$. The PAR3 mitogenome sequence will provide a useful reference for comparing different Trichoderma strains from the United States and around the world.

Fungal strain PAR3 was isolated from roots of a Scarlet Royal grapevine on Freedom rootstock growing in a vineyard in Parlier, CA, U.S.A., in the summer of 2020. Morphological identification of PAR3 suggested it was $T$. harzianum Rifai (which has the teleomorph Hypocrea lixii Pat. according to Chaverri and Samuels 2002), following a dichotomous key by Chaverri and Samuels (2003) and Chaverri et al. (2016). As a strain of T. harzianum, PAR3 exhibits putative activity against various fungal pathogens, including those that could cause grapevine trunk diseases such as Diplodia seriata, Eutypa lata, and Neofusicoccum parvum. All of these pathogens cause cankers that develop over time, ultimately resulting in the need to replant vineyards every 10 to 12 years where prevalent (Baumgartner et al. 2019; Kaplan et al. 2016). Thus, there exists a need to further characterize PAR3.

Mitochondrial genomes are important in species identification and marker development due to small sizes, high copy numbers, and presence of both conserved and variable regions (Medina et al. 2020). Previously, a variety of Trichoderma spp. have had mitochondrial genomes reported and analyzed (Kwak 2020). However, only a handful of species, consistently mostly of industry-useful isolates, have been analyzed by Kwak (2020). Therefore, the PAR3 mitochondrial genome as a resource is an important addition to expand our knowledge of Trichoderma species.

Total genomic DNA was extracted using a Macherey-Nagel (Bethlehem, PA, U.S.A.) Plant Mini Kit from 1-week-old PAR3 cultures grown in potato dextrose broth (Difco Laboratories,

†Corresponding author: C. M. Wallis; christopher.wallis@ars.usda.gov

Data availability: The PAR3 mitogenome sequence has been deposited at DDBJ/EMBL/GenBank under accession number MZ713368.

The author(s) declare no conflict of interest.

Accepted for publication 17 October 2021.

\section{Keywords}

biological control agents, grapevine, Hypocrea lixii, mitochondrial genome 
A

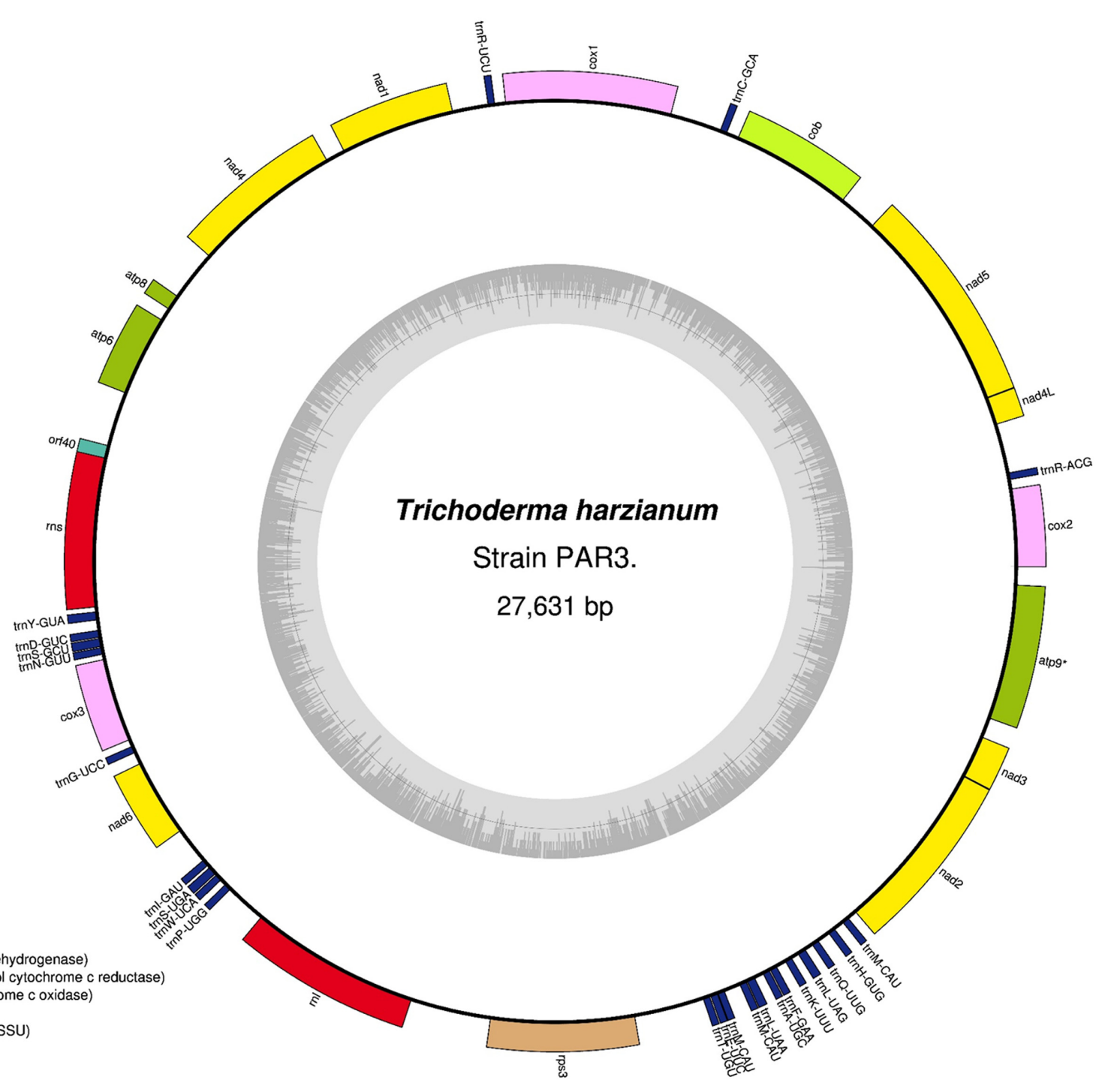

B

complex I (NADH dehydrogenase)

complex III (ubichinol cytochrome $\mathrm{c}$ reductase)

complex IV (cytochrome coxidase)

ATP synthase

ribosomal proteins (SSU)

ORFs

ransfer RNAs

ribosomal RNAs

MT108299.1 Cladobotryum mycophilum

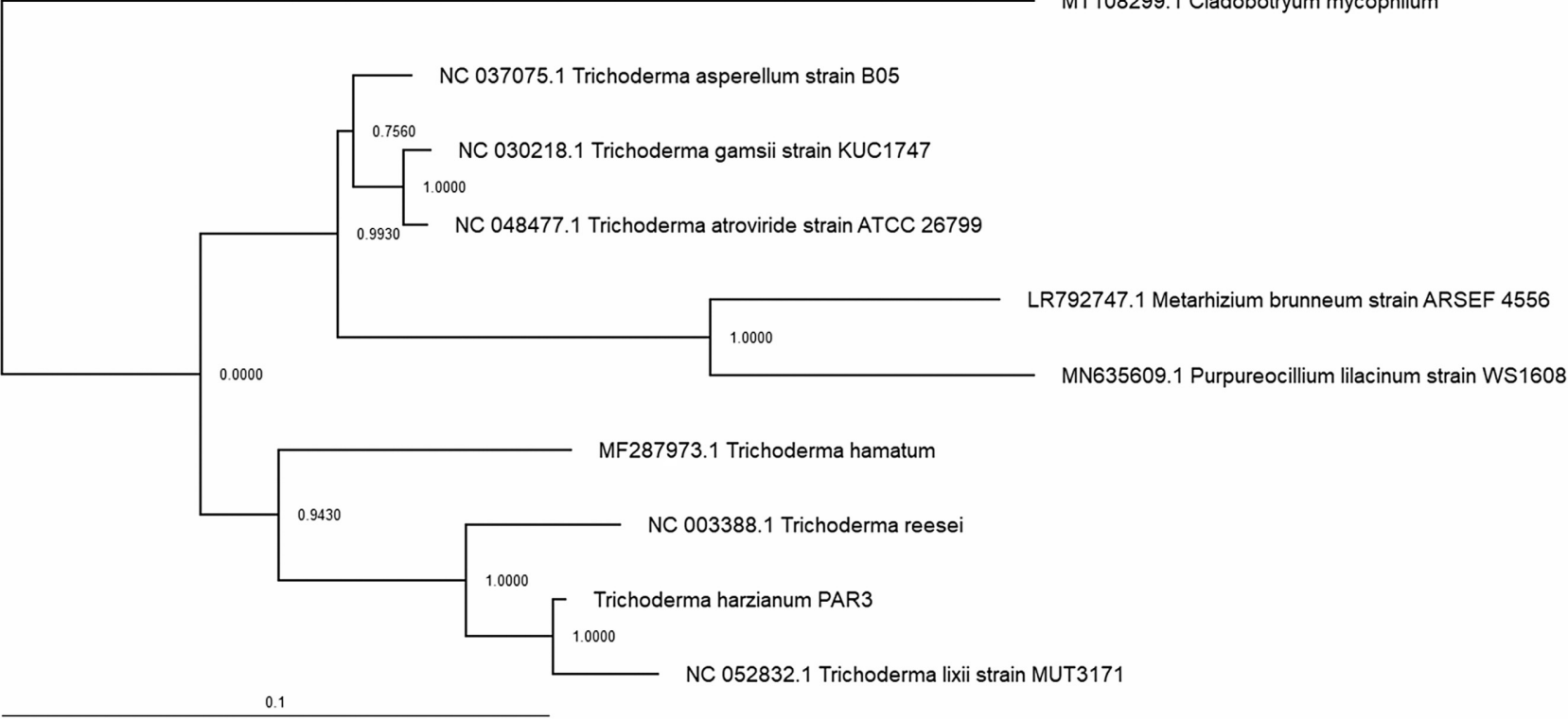

Fig. 1. A, A genomic map of the mitogenome of Trichoderma harzianum Strain PAR3. Figure was made using OrganellarGenomeDRAW (OGDRAW) version 1.3.1 (Greiner et al. 2019). B, A phylogenetic tree showing the placement of Trichoderma harzianum strain PAR3 mitogenome referenced to closely related sequences currently available in the GenBank database. 
Table 1. Nucleotide variations of mitogenome sequences between Trichoderma lixii strain MUT3171 (NC_052832.1/MT495248.1, 29,791 bp) and T. harzianum PAR3 (MZ713368.1, 27,631bp)

\begin{tabular}{lcc} 
Position (NC_052832.1) & MUT3171 & PAR3 \\
188 & - & A \\
794 & - & A \\
795 & - & T \\
$1,544-2,883$ & $1,339 \mathrm{bp}$ & - \\
$13,417-14,238$ & $821 \mathrm{bp}$ & - \\
13,413 & $\mathrm{G}$ & $\mathrm{A}$ \\
13,414 & $\mathrm{~T}$ & $\mathrm{C}$ \\
13,415 & $\mathrm{C}$ & $\mathrm{T}$ \\
13,416 & $\mathrm{C}$ & $\mathrm{T}$ \\
15,406 & $\mathrm{~T}$ & $\mathrm{C}$ \\
17,536 & $\mathrm{~A}$ & $\mathrm{C}$ \\
18,743 & $\mathrm{G}$ & $\mathrm{A}$ \\
19,127 & $\mathrm{~T}$ & $\mathrm{~A}$ \\
20,330 & $\mathrm{C}$ & $\mathrm{T}$ \\
28,305 & $\mathrm{~A}$ & - \\
\hline
\end{tabular}

Detroit, MI, U.S.A.) on a shaker under ambient light and at $26^{\circ} \mathrm{C}$. An Invitrogen (Carlsbad, CA, U.S.A.) Qubit fluorometer was used to assess DNA quantity and quality. Afterward, the genomic DNA was sequenced using an Illumina HiSeq $2 \times 100$ paired-end format (Illumina, San Diego, CA, U.S.A.) through a commercial source after the amount of DNA was increased using Illustra GenomiPhi version 2 DNA amplification kits (GE Healthcare, Waukesha, WI, U.S.A.).

The HiSeq sequencing yielded a total of $52,337,510,850$ bp with a read size of 100 bp and a $Q$ value greater than 30 . The short reads were assembled de novo using the CLC genomics workbench (version 10.0). This resulted in 44,766,176 bp in 1,723 contigs $(>1,000 \mathrm{bp})$. With the help of BLAST searches, a 645 -bp internal transcribed spacer (ITS) sequence was identified using the sequences of primer ITS4 (TCCTCCGCTTATTGATATGC) and ITS5 (GGAAGTAAAAGTCGTAACAAGG) (Chaverri et al. 2016). A 339-bp translation elongation factor 1-alpha (TEF1) sequence was identified using the sequences of primers EF1-728F (CATCGAGAAGTTCGAGAAGG) and EF1-986R (TACTTGAAGGAACCCTTACC) (Chaverri et al. 2016). A BLAST search against the GenBank database using both ITS and TEF1 sequences confirmed that PAR3 was a strain of $T$. harzianum.

Referenced to MT495248.1, a contig of 27,813 bp was identified through a BLASTn search. After manually editing overlaps at the $5^{\prime}$ and $3^{\prime}$ ends, a sequence of 27,631 bp was collected and designated as the HiSeq version of the PAR3 mitogenome. For further confirmation of the mitogenome structure, the PAR3 DNA was also sequenced using a minION system from Oxford Nanopore (Alameda, CA, U.S.A.). Nanopore sequencing yielded a total of $1,057,994,148 \mathrm{bp}$ sequences with $516,174,469 \mathrm{bp}$ (140,849 reads) ranging from 1,000 to 50,000 bp with a $Q$ value greater than 20 . Sequence reads were assembled with Canu software (version 2.1) (Koren et al. 2017). A total of 7,636,456 bp sequences were assembled with 446 contigs. A circular 43,401-bp contig was identified and trimmed to $27,549 \mathrm{bp}$ after overlap removal; it was designated as the Nanopore version of the PAR3 mitogenome. The HiSeq version (27,631 bp) and Nanopore version (27,549 bp) were compared through BLASTn (word size $=7$ ). The two versions were identical in sequence order and over $99 \%$ similar in nucleotides. The difference of 82 bp $(27,631-27,549=82)$ was likely due to nucleotide sequence errors mostly in the Nanopore versions. Because HiSeq sequences have higher $Q$ values than Nanopore sequences ( 30 versus 20 , respectively), the HiSeq version was selected as T. harzianum strain PAR3 mitogenome version 1. Annotation of the mitogenome sequence was performed using GeSeq (Tillich et al. 2017).

The finalized PAR3 mitogenome is $27,631 \mathrm{bp}$, consisting of 14 core mitochondrial protein genes coding for adenosine triphosphate synthases (apt), apocytochrome b (cob), cytochrome $c$ oxidases (cox), and nicotinamide adenine dinucleotide hydrogen (NADH) dehydrogenases (nad); a ribosomal protein (rps); 25 transfer RNAs; and two ribosomal RNAs (Fig. 1A). There was one additional open reading frame (orf40) annotated as an unknown putative protein. All genes were located on the heavy strand ( $\mathrm{H}$-strand). The overall composition of the complete mitochondrial genome had a GC\% content of $27.55 \%$. 
A BLASTn search using the PAR3 mitogenome sequence as a query against the GenBank nr database showed extensive matches (>99\% query coverage and $99.72 \%$ identity) to the mitogenome sequence of $T$. lixii strain MUT3171 (MT495248.1, 29,791 bp) reported from Italy (Venice et al. 2020). Table 1 shows the nucleotide variations including two major deletion events between the two mitogenomes identified using the CLUSTAL program (McWilliam et al. 2013).

For phylogenetic analysis, the mitogenome sequences of six Trichoderma species and three related fungal species identified through the BLAST search were downloaded from the GenBank database. Together with the PAR3 mitogenome, a phylogenetic tree was constructed using MEGA (version 6) with the maximum likelihood method and a bootstrap replication number of 500 (Fig. 1B).

The mitochondrial genome of the T. harzianum PAR3 strain will enable further comparisons with those of other Trichoderma spp., facilitating future identification of useful members of that species for use in biological control and bioremediation, as well as for other purposes.

\section{Acknowledgments}

The authors thank Nalong Mekdara and Yadira Andrade for their technical assistance in this work. Mention of trade names or commercial products in this publication is solely for the purpose of providing specific information and does not imply recommendation or endorsement by the U.S. Department of Agriculture. USDA is an equal opportunity provider and employer.

\section{Literature Cited}

Baumgartner, K., Hillis, V., Lubell, M., Norton, M., and Kaplan, J. 2019. Managing grapevine trunk diseases in California's Southern San Joaquin Valley. Am. J. Enol. Vitic. 70:267-276.

Chaverri, P., Branco-Rocha, F., Jaklitsch, W., Gazis, R., Degenkolb, T., and Samuels, G. J. 2016. Systematics of the Trichoderma harzianum species complex and the re-identification of commercial biocontrol strains. Mycologia 107:558-590.

Chaverri, P., and Samuels, G. J. 2002. Hypocrea lixii Pat., the teleomorph of Trichoderma harzianum Rifai. Mycological Prog. 1:283-286.

Chaverri, P., and Samuels, G. J. 2003. Hypocrea/Trichoderma (Ascomycota, Hypocreales, Hypocreaceae): Species with green ascospores. Stud. Mycol. 48:1-116.

Greiner, S., Lehwark, P., and Bock, R. 2019. OrganellarGenomeDRAW (OGDRAW) version 1.3.1: Expanded toolkit for the graphical visualization of organellar genomes. Nucleic Acids Res. 47:59-64.

Kaplan, J., Travadon, R., Cooper, M., Hillis, V., Lubell, M., and Baumgartner, K. 2016. Identifying economic hurdles to early adoption of preventative practices: The case of trunk diseases in California winegrape vineyards. Wine Econ. Policy 5: 127-141.
Koren, S., Walenz, B. P., Berlin, K., Miller, J. R., and Phillippy, A. M. 2017. Canu: Scalable and accurate long-read assembly via adaptive k-mer weighting and repeat separation. Genome Res. 27:722-736.

Kwak, Y. 2020. Complete mitochondrial genome of the fungal biocontrol agent Trichoderma atroviride: Genomic features, comparative analysis and insight into the mitochondrial evolution in Trichoderma. Front. Microbiol. 11:785.

McWilliam, H., Li, W., Uludag, M., Squizzato, S., Park, Y. M., Buso, N., Cowley, A. P., and Lopez, R. 2013. Analysis tool web services from the EMBL-EBI. Nucleic Acids Res. 41:W597-W600 doi: 10.1093/nar/gkt376.

Medina, R., Franco, M. E. E., Bartel, L. C., Martinez Alcántara, V., Saparrat, M. C. N., and Balatti, P. A. 2020. Fungal mitogenomes: Relevant features to planning plant disease management. Front. Microbiol. 11:978.

Tillich, M., Lehwark, P., Pellizzer, T., Ulbricht-Jones, E. S., Fischer, A., Bock, R., and Greiner, S. 2017. GeSeq - Versatile and accurate annotation of organelle genomes. Nucleic Acids Res. 45:W6-W11.

Venice, F., Davolos, D., Spina, F., Poli, A., Prigione, V. P., Varese, G. C., and Ghignone, S. 2020. Genome sequence of Trichoderma lixii MUT3171, a promising strain for mycoremediation of PAH-contaminated sites. Microorganisms 8:1258. 